\title{
BMJ Open Efficacy and safety of a supplement combination for hand osteoarthritis pain: protocol for an internet-based randomised placebo-controlled trial (The RADIANT study)
}

Xiaoqian Liu (1) , ${ }^{1}$ Sarah Robbins, ${ }^{1}$ Jillian Eyles, ${ }^{1}$ Tatyana Fedorova, ${ }^{1}$ Sonika Virk, ${ }^{1}$ Leticia A Deveza, ${ }^{1}$ Andrew McLachlan, ${ }^{2}$ David Hunter (i) ${ }^{1}$

To cite: Liu X, Robbins S, Eyles J, et al. Efficacy and safety of a supplement combination for hand osteoarthritis pain: protocol for an internet-based randomised placebo-controlled trial (The RADIANT study). BMJ Open 2020;10:e035672. doi:10.1136/ bmjopen-2019-035672

- Prepublication history for this paper is available online. To view these files, please visit the journal online (http://dx.doi. org/10.1136/bmjopen-2019035672).

Received 11 November 2019

Revised 29 January 2020

Accepted 30 January 2020

Check for updates

(C) Author(s) (or their employer(s)) 2020. Re-use permitted under CC BY-NC. No commercial re-use. See rights and permissions. Published by BMJ.

${ }^{1}$ Northern Clinical School, Institute of Bone and Joint Research, Kolling Institute of Medical Research, Rheumatology Department, Royal North Shore Hospital, The University of Sydney Faculty of Medicine and Health, Sydney, New South Wales, Australia

${ }^{2}$ School of Pharmacy, The

University of Sydney Faculty of Medicine and Health, Sydney, New South Wales, Australia

Correspondence to Professor David Hunter; david.hunter@sydney.edu.au

\section{ABSTRACT}

Introduction Hand osteoarthritis (HOA) is a highly prevalent disabling joint disease. The current management regimens are limited. Potentially as a consequence, many people turn to complementary and alternative medicines for symptomatic relief. A combination of two or more supplements is common in clinical practice; however, evidence for the efficacy of this approach is lacking. The aim of this study is to investigate the efficacy of a supplement combination for treating symptomatic HOA in comparison to placebo.

Methods and analysis The RADIANT study is an internetbased, parallel, superiority, double-blind, placebo-controlled, randomised, two-arm clinical trial. A participatory design is used to facilitate the study procedures. One hundred and six participants aged over 40 years with painful HOA and structural change on X-ray (Kellgren and Lawrence grade $(K L G) \geq 2$ ) will be recruited from the community and randomly allocated to receive either a supplement combination composed of: (1) combined supplement containing Boswellia serrata extract, pine bark extract and methylsulfonylmethane and (2) curcumin or placebo for 12 weeks. The primary outcome will be 12-week change in hand pain on a visual analogue scale (VAS). Main secondary outcomes include adverse events, change in hand function, patient global assessment of disease activity and quality of life. A range of additional measures will be recorded, and an individual patient placebo response will be performed. The primary analysis will be conducted using an intentionto-treat approach. Adverse events will be monitored weekly throughout the study.

Ethics and dissemination This protocol has been approved by the University of Sydney Human Research Ethics Committee (HREC No. 2018/766). Dissemination will occur through conferences, social media, scientific publications and $\mathrm{PhD}$ thesis.

Trial registration number Australian New Zealand Clinical Trials Registry (ACTRN12619000835145); Preresults

\section{INTRODUCTION}

\section{Background and rationale}

Osteoarthritis (OA) is a highly prevalent, chronic and disabling joint disease, leading

\section{Strengths and limitations of this study}

- The RADIANT study will use evidence from a systematic review to propose a novel complementary medicine to evaluate efficacy and safety in hand osteoarthritis.

- This will be an internet-based study that uses online recruitment and data capture without any hospital visits which will decrease participants travel burden and increase the recruitment rate in an efficient and cost-effective way.

- Participants have been involved in the study design. This will help identify logistical issues to facilitate adherence to the study.

- Participants recruited from an online study may not represent a similar population as to a sample in the clinical practice.

- A limitation of this trial is that, if the combination complementary medicine is beneficial, we still will not know which of the four supplements are the most effective ingredients.

to pain and functional limitations with high social and economic costs. ${ }^{1}$ The impact of OA and joint pain is set to rise as the global ageing population continues to grow. ${ }^{2}$ The hand is the most commonly affected site. The prevalence of hand OA (HOA) demonstrates wideranging estimates due to differences in disease definitions, types of populations and/or risk factors. $^{3-5}$

HOA is a heterogeneous disorder affecting multiple joints with various presentations and several phenotypes including thumb base $\mathrm{OA}$, interphalangeal (IP) $\mathrm{OA}$ and erosive OA. Among those, erosive HOA is associated with the most severe clinical symptoms and the most disability. ${ }^{6}$ Patients with HOA frequently report hand pain, stiffness, deformity and frustration in undertaking everyday activities such as opening jars, carrying weights and writing. ${ }^{7}$ 
HOA can be diagnosed from radiographic and clinical perspectives. ${ }^{8}$ The American College of Rheumatology (ACR) classification criteria of HOA is the most widely used validated clinical diagnostic criteria, although it has the limitation without definition for thumb base OA. ${ }^{6}$

The current recommendations of management strategies for HOA include non-pharmacological and pharmacological treatment modalities (ie, limited duration of analgesics particularly non-steroidal anti-inflammatory drugs (NSAIDs)) targeting symptom alleviation (eg, pain reduction) $;{ }^{9}{ }^{10}$ however, high-quality trials are still scarce. Due to the limited management regimens available, many people turn to complementary and alternative medicines (CAMs) for symptomatic relief. ${ }^{11} 12$ The prevalence of CAMs use varies from $40 \%$ to $69 \%$ depending on the population studied. ${ }^{1314}$ The most common CAMs consumed by people with OA include fish, krill or omega oil $(74 \%)$ and glucosamine $(54 \%) .{ }^{14}$ The average annual per-person expenditures for alternative and traditional therapies are almost the same at US $\$ 1127$ versus US $\$ 1148$, respectively. ${ }^{15}$ Therefore, it is important to consider the evidence for the efficacy of these medicines.

A systematic review that investigated the efficacy and safety of dietary supplements for treating OA found that some of the most widely used supplements such as glucosamine and chondroitin were not clinically effective in short-term. Lesser-known supplements Boswellia serrata extract (BSE, effect size -1.61 ( -2.1 to -1.13$)$ ), pine bark extract (PBE, effect size $-1.21(-1.53$ to -0.89$)$ ), curcumin (effect size -1.19 (-1.93 to -0.45$)$ ) and methylsulfonylmethane (MSM, effect size $-1.10(-1.81$ to -0.38$)$ ) were identified as the CAMs with the largest treatment effects for symptomatic relief in people with OA, although trial quality was a concern. ${ }^{16}$ To date, we believe that there is no new evidence that would challenge the results of this systematic review. The overall treatment effects of individual complementary medicines are modest. However, combining supplements with similar pharmacological effects has the potential to afford larger cumulative effects. Although there is a paucity of robust research evidence to support the efficacy of the combinations, in practice a combination of two or even more than three supplements is commonly consumed by people with HOA. ${ }^{14}$

BSE, PBE, curcumin and MSM are generally recognised as safe. ${ }^{17}$ BSE has been shown to cause minor reactions like nausea or a headache in some people. Curcumin can cause hypotension. There might be a potential interaction between curcumin and anticoagulant medications. ${ }^{17}$ There have not been any major adverse effects reported regarding PBE and MSM. Given the favourable safety profile of these CAMs, the products of this study were deemed to be safe to use. Moreover, a phase 1 pharmacokinetic study was conducted prior to this study and found that the supplement combination of these medicines could be investigated to manage patients with $\mathrm{OA}$ without significant concerns for possible pharmacokinetic interactions. ${ }^{18}$ No adverse events (AEs) were reported in the pharmacokinetic study. ${ }^{18}$ Therefore, the aim of this study is to investigate the efficacy of the supplement combination containing BSE, PBE, MSM and curcumin for treating symptomatic HOA in comparison to placebo.

Web-based technology is increasingly used to conduct clinical trials due to cost-effectiveness, convenience and attractiveness to participants which results in enhanced recruitment rate. ${ }^{19}{ }^{20}$ An internet-based clinical trial of glucosamine was successfully conducted in $2004 .^{21}$ This study will be conducted using an internet-based trial design without the need for hospital or clinician-based recruitment and review. This is acceptable in view of the adequate safety profile of the supplements and the ability to communicate with participants online, over the phone and view imaging and tests remotely. To increase the chances of operational success, a participatory design process will be used in the early design to facilitate online surveys and test study procedures. ${ }^{22} 23$

\section{Objectives}

The primary objective is to determine the efficacy of the supplement combination for treating symptomatic HOA based on patient-reported hand pain. The secondary objective is to determine the safety and efficacy of the supplement combination based on AEs, patient-reported hand function, health-related quality of life and patient global assessment (PGA) of disease activity.

\section{METHODS AND ANALYSIS \\ Study design}

This protocol is described using the 2013 Standard Protocol Items: Recommendations for Interventional Trials (SPIRIT) guidelines on standard protocol items for clinical trials. ${ }^{24}$ The RADIANT study is designed as an internet-based, 12-week, parallel, superiority, doubleblind (participants and investigators will be blinded), placebo-controlled, randomised, two-arm clinical trial with 1:1 allocation ratio.

\section{Study setting}

The RADIANT study will be conducted online through Research Electronic Data Capture (REDCap). The study will be coordinated by the Institute of Bone and Joint Research (IBJR), Kolling Institute of Medical Research, The University of Sydney, New South Wales, Australia. The investigational products will be stored, dispensed and posted by PCI Pharma Services (Port Melbourne, Victoria, Australia).

\section{Participant recruitment and eligibility criteria}

One hundred and six participants (53 in each arm) with the evidence of HOA at least in the most painful hand (index hand) will be recruited from the community and research volunteer database. The recruitment strategies will include (1) advertisements on social media networks (eg, Facebook, Twitter) and the IBJR website, (2) emailed advertisements to the IBJR research volunteer database and the Bod Australia patients' database, (3) posters/flyers placed 
on medical practices and community areas, (4) foyer events at Royal North Shore Hospital and (5) radio interview.

Participants will be eligible for the study if they meet all inclusion criteria below:

1. Functional English, internet access and an active email account.

2. $\geq 40$ years-old, male or female, Australian permanent resident.

3. Hand pain for at least half of the days in the previous month.

4. Hand pain $\geq 40$ and $\leq 90$ out of 100 on the VAS over the last week.

5. The functional index for hand osteoarthritis (FIHOA) scores $\geq 6$ out of 30 .

6. Clinical diagnosis of HOA according to ACR classification criteria which will be assessed by visual observation of hand photographs taken by patient themselves, ${ }^{25}$ and/or hand pain at thumb base.

7. Radiographic evidence of HOA (KLG $\geq 2)$ in at least one of the painful joints; and/or central erosions in at least one IP joint. ${ }^{6}$ KLG is a common method of classifying the severity of $\mathrm{OA}$ using five grades from grade 0 (no radiographic features of $\mathrm{OA}$ ) to grade 4 (large osteophytes, marked joint space narrowing, severe sclerosis and definite bony deformity). ${ }^{26}$

Participants will be excluded if they meet any of the following criteria:

1. Unable to be reached after screening survey and before being enrolled.

2. Pregnant or breast feeding, childbearing potential but not willing to use contraceptive methods for the duration of the study.

3. Crystal-related arthritis (eg, gout), autoimmune arthritis (eg, rheumatoid arthritis, psoriatic arthritis, systemic lupus erythematosus), hemochromatosis or fibromyalgia.

4. Painful syndrome of the upper limb (eg, carpal/cubital tunnel syndrome).

5. Hand injury in the past 6 months.

6. Any clinically significant acute or ongoing chronic medical conditions that could compromise patient safety, limit the patient's ability to complete the study and/or compromise the objectives of the study. The list of excluded health conditions is presented in box 1.

7. Currently taking medications known to have potential pharmacological interactions with the study supplements (eg, anticoagulant medications).

8. Allergic to ingredients of the study supplements.

9. Taking centrally acting analgesics (eg, opioid) regularly.

10. Taking NSAIDs (oral or topical) but unable to undergo a 1-week washout and/or not willing to stop it for the duration of the study.

11. Taking centrally acting analgesics occasionally but unable to undergo a 1week washout and/or not willing to stop it for the duration of the study.

12. Taking any concomitant supplements.
Box 1 List of excluded health conditions

\section{Health conditions}

- Ischaemic heart disease/coronary heart disease, heart valves disorders or arrhythmia (irregular heartbeats; follow-up question to assure that condition is stable).

- Cancer (follow-up question to include participants with at least 3 years of cancer-free).

- Stroke/cerebrovascular disorders, tumour of the brain or arteriovenous malformation (follow-up question to assure that condition is stable).

- Uncontrolled diabetes.

Renal failure or renal dialysis.

- Chronic liver disorders, liver cirrhosis, hepatic encephalopathy or liver failure.

- AIDS/HIV infection.

- Dementia/Alzheimer's disease.

- Severe psychiatric or mental illness.

Motor neuron disease.

Muscular dystrophy.

- Paralysis (hemiplegia/paraplegia/quadriplegia).

- Chronic lung disease.

- Multiple sclerosis.

- Parkinson disease with complications.

- Pulmonary hypertension.

Aplastic anaemia, thalassaemia major or severe blood disorders.

13. Surgery in the index hand in the last 12 months or surgery plan in the next 6 months.

14. Intra-articular (IA) hyaluronic acid injection in the past 6 months, IA corticosteroid in the past 3 months or IA autologous blood product in the past 12 months.

15. Participation in another clinical trial and/or treatment received with any investigational products within 30 days before enrolment.

\section{Interventions}

The active treatment group will receive a supplement combination composed of: (1) combined supplement containing BSE (Boswellin Super) $250 \mathrm{mg} /$ day, PBE (Fenoprolic 70 Organic) $100 \mathrm{mg} /$ day and MSM $1500 \mathrm{mg} /$ day and (2) curcumin (Flexofytol) $168 \mathrm{mg} /$ day. The placebo group will receive: (1) placebo-combined supplement and (2) placebo curcumin. Table 1 provides details of the interventions. The product material of BSE, PBE and MSM will be sourced in bulk powder and curcumin (including placebo curcumin) will be sourced in bulk capsules from Bod Australia (Double Bay, New South Wales, Australia). The material for the placebo-combined supplement will be provided by PCI Pharma Services. The daily dose of the combined supplement will be encapsulated in three size 00 light blue opaque gelatin capsules. The placebo-combined supplement will be manufactured to have the same size and appearance. PCI Pharma Services will be responsible for the encapsulation, labelling and dispensing of the study products.

All products will be manufactured according to Good Manufacturing Practice (GMP) as per the International Conference on Harmonisation (ICH) Quality Guidelines. 


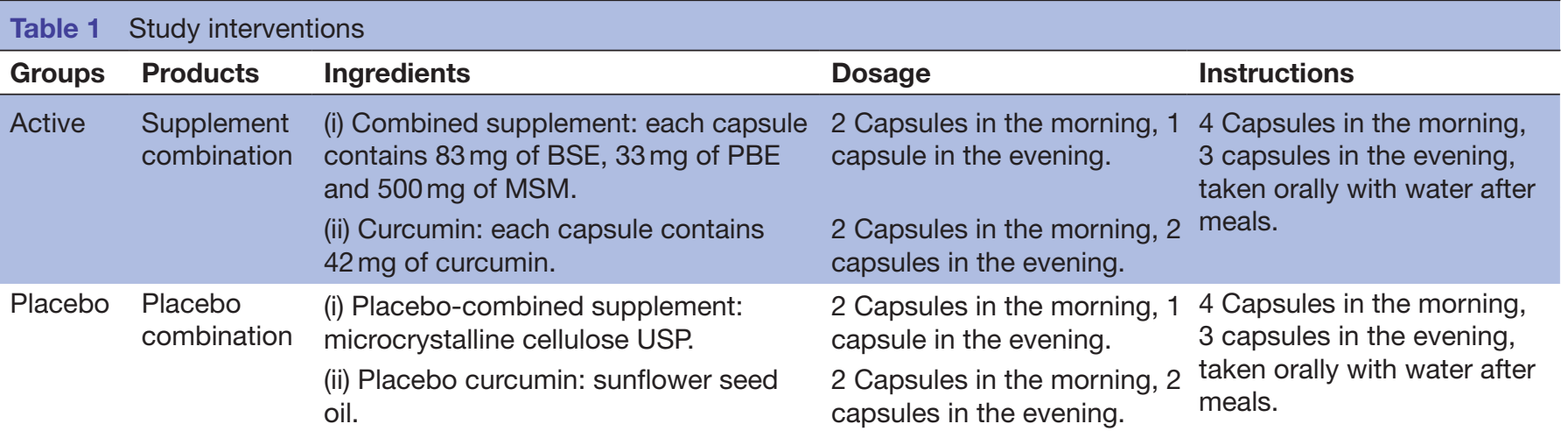

BSE, Boswellia serrata extract; MSM, methylsulfonylmethane; PBE, pine bark extract; USP, United States Pharmacopoeia.

The label will comply with the Therapeutic Goods Administration requirements for clinical trials. The study kits for each participant containing enough supplements for 12-week treatment will be prepared in batches and stored at the warehouse of PCI Pharma Services. Emergency study kits containing supplements for 4-week treatment will be allowed for any replacements of misplaced products.

\section{Randomisation, allocation and blinding}

Participants will be randomly assigned to either active or placebo group with a 1:1 allocation rate as per computergenerated randomisation, scheduled using random permuted block sizes and stratified by erosive or nonerosive HOA.

The sequence generation will be prepared by a statistician not involved in the study. The allocation will be concealed from all investigators in sequentially numbered opaque, sealed and stapled envelopes. Aluminium foil inside the envelope will be used to render the envelope impermeable to intense light. Envelopes will be kept in a locked drawer of an unblinded researcher and will be opened after the baseline survey is returned completely.

The unblinded researcher will inform PCI Pharma Services participants' allocation for dispensing and shipment. One unblinded staff in PCI Pharma Services will prepare the study kits. The staff responsible for dispensing will be blinded.

Participants will be blinded to their treatment allocation. The active and placebo products will be provided in identical appearance and size to ensure appropriate blinding. There will be a code-breaking list available in case of serious AEs which will be held by the unblinded researcher. Participants will have their group allocation revealed automatically after the week 12 survey.

\section{Concomitant medication}

Participants will be asked to discontinue NSAIDs, analgesics and other supplements for hand pain, with the exception of paracetamol (maximum $3000 \mathrm{mg} /$ day) for rescue pain relief from 1 week before baseline assessment until 12-week follow-up assessment. All concomitant medication will be recorded.

\section{Outcome measures}

The outcome measures will be patient-reported outcomes in their index hand, where appropriate with good reliability, validity and responsiveness. ${ }^{27}$ Participants will be asked to choose which their most painful hand is, this will be the index hand. Study outcome measures are presented in table 2 . The primary outcome will be:

1. Change in hand pain: measured from baseline to week 12 using a VAS from 0 (no pain) to 100 (worst pain possible) with the question 'How much pain in your hand did you experience on average over the last week? Please consider the pain felt while using your hand during daily activities'. There is evidence of good construct validity and some evidence for responsiveness and discrimination for the use of pain VAS in participants with HOA.

The secondary outcomes include:

1. AEs: assessed by inspection of weekly surveys.

2. Change in hand pain: measured from baseline to week 2 and 6 using VAS (0-100).

3. Change in hand function: measured from baseline to weeks 2, 6 and 12 using FIHOA. FIHOA is a questionnaire either physician-administered or self (patient)administered, made up of 10 questions scored according to a 4-grade scale. The total score ranges from 0 (no functional impairment) to 30 points (maximal impairment), with a validated threshold of 5 , able to distinguish between symptomatic and not symptomatic patients. ${ }^{27}$

4. Change in PGA of disease activity: measured from baseline to weeks 2, 6 and 12 using a VAS scale from 0 (very well) to 100 (very poor) with the question 'Considering all the ways your HOA affects you, how have you been during the last 48 hours? 28

5. Change in health-related quality of life: measured from baseline to weeks 2, 6 and 12 using assessment of quality of life four dimension (AqoL-4D) which covers four domains of independent living, mental health, relationships and senses with a total of 12 items and 3 items per dimension. The AQoL-4D items have four numbers of response levels. Response options are on 


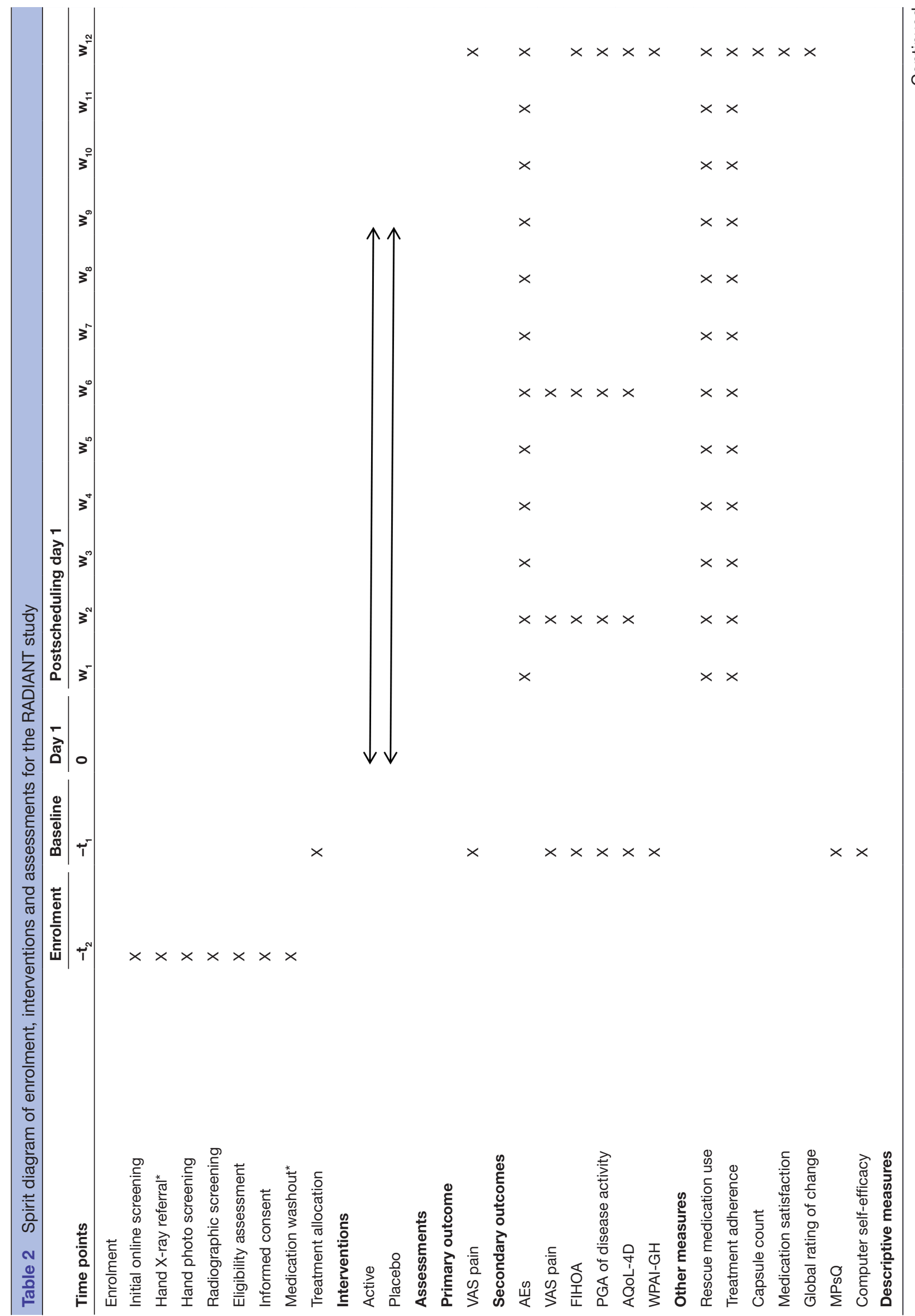


a Guttman scale, with higher scores indicative of progressively higher levels of disability. ${ }^{29}$

6. Change in impairments in work and activities: measured from baseline to week 12 using the Work Productivity and Activity Impairment questionnaire general health version which consists of six questions: $1=$ currently employed; $2=$ hours missed due to health problems; $3=$ hours missed other reasons; $4=$ hours actually worked; $5=$ degree health affected productivity while working (using a $0-10$ VAS); $6=$ degree health affected productivity in regular unpaid activities (VAS). The recall period for the questions 2 to 6 is 7 days. ${ }^{30}$

Other measures include:

1. Consumption of rescue medication: measured by inspection of weekly surveys.

2. Treatment adherence: defined as how much medication participants are consuming and measured weekly by the self-reported non-adherence questionnaire ${ }^{31}$ including three items using a 5-grade scale and two comprehensive questions regarding the number of doses missed and the reason. Patient-reported capsule count will be collected at the end of the study.

3. Patient satisfaction with allocated treatment: assessed at the end of the study using the Medication Satisfaction Questionnaire $^{32}$ with two questions 'Overall, how satisfied are you with the current supplement(s) for your hand osteoarthritis?' with a 7-point Likert scale from extremely dissatisfied to extremely satisfied and 'How would you describe your hand PAIN intensity since you began the study?' with a 5-point Likert scale from much worse to much better.

4. Global rating of change ${ }^{33}$ for pain and function: assessed at the end of the study using a 5-point Likert scale ranging from much better to much worse with the question 'Which option best represents the change in pain/ change in function in your hand since you began the study?'

5. Individual patient placebo response: assessed at baseline using the Multidimensional Personality Questionnaire $(\mathrm{MPsQ})^{34} 35$ which is a self-reported questionnaire using a 5-point scale ranging from 1 (strongly disagree) to 5 (strongly agree) contributing to the evaluation of individual patient response to placebo by assessing patients' personality, well-being as well as attitudes and beliefs on disease therapies.

6. Participants' technology self-efficacy: measured at baseline using a modified computer self-efficacy scale with 10 items according to a 10 -grade scale. ${ }^{36}$

\section{Patient and public involvement}

Patients and the public were not directly involved in the development of the research question. However, patients suffering from HOA will be involved in the study design and conduct by participating in a small pilot study which will take place before study initiation. Three patients will be included in the pilot study to assess the study procedures, the burden of the intervention and time required to participate in the research. These patients will review 


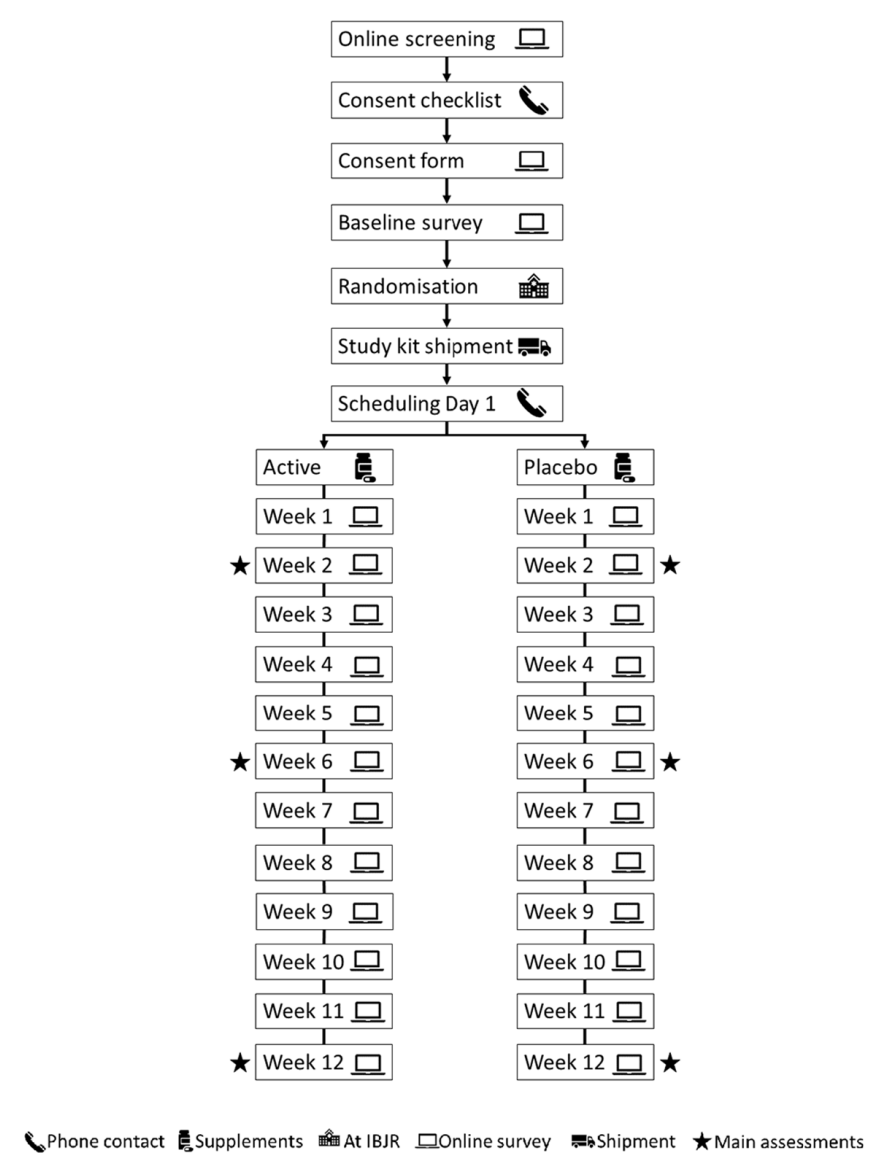

Figure 1 Flow diagram of the study protocol. IBJR, Institute of Bone and Joint Research.

relevant documents and provide feedback on them. The data collected from these participants will not be used in the main trial. At the conclusion of the study, overall study findings will be provided to study participants in lay summary.

\section{Study procedures}

The sequence of study events and the schedule for enrolment, screening, interventions and assessments are summarised in figure 1 and table 2.

\section{Screening}

Participants will complete an online survey to determine initial eligibility. The clinical and radiographic diagnosis of HOA will be assessed using digital hand photographs (both hands) and radiographs (index hand). ${ }^{37} 38$ Potentially eligible participants will be instructed to provide a photograph of their both hands for clinical assessment using ACR criteria. ${ }^{25}$ Participants will provide hard copies or permission to access their hand X-ray taken within the last 36 months (this time interval was chosen with the justification of the evidence that only $20 \%-25 \%$ of people will actually demonstrate progression on KLG during this interval). ${ }^{39}$ Individuals without an X-ray within 36 months will be referred to have an X-ray taken. The posterior-anterior view will be used to assess eligibility. HOA severity will be assessed using KLG and erosive OA will be assessed using Osteoarthritis Research Society International (OARSI) atlas. ${ }^{40}$ Both the hand photograph and $\mathrm{xX}$-ray will be assessed by a trained rheumatologist.

\section{Informed consent process}

Participants who are deemed eligible will be informed of the research project over the phone and electronically sign the consent form using the REDCap e-Consent Framework. Those participants referred to have an x-ray will provide written informed consent before receiving the referral.

\section{Pretrial washout}

Participants who are taking NSAIDs, analgesics and/or supplements not containing study products will undergo 1 week washout before baseline assessment. Participants who are taking supplements containing any ingredients of the study products will undergo 2 months washout before baseline assessment.

\section{Enrolment}

Eligible participants will then complete the baseline survey. Demographic data, medical history, primary and secondary outcomes, MPsQ and modified computer self-efficacy scale will be collected. After the baseline survey is completed and returned, participants will be assigned a study enrolment number and this number will be recorded in all participant-specific study documents. Enrolled participants will be sent Participant's Information Booklet, Participant's Identification Card and Participant's Instructions.

\section{Study kit dispensing, shipment and scheduling Day 1}

A dispensing log will be created and regularly updated by the unblinded researcher based on the packaging slip received from PCI Pharma Services. Day 1 will be scheduled over the phone once participants receive the study kits which will be anticipated within 7-10 days after baseline survey.

\section{Follow-up surveys}

Treatment adherence, AEs and the use of rescue medication will be assessed weekly. The primary and secondary outcomes will be assessed at weeks 2, 6 and 12 and participants will not be allowed to take rescue medication over the week before each of these surveys. At the week 12 survey, participants will also be asked about their satisfaction with the treatment received and to provide capsule counts.

\section{Adverse events}

The risks for participants involved in this study are minimal. Any potential AEs will be monitored and assessed through weekly surveys during the research period. This will include questions about any AEs that participants experienced including their nature, how long they lasted for and what action if any, they took (eg, taking medication or seeing a health professional). The study physician will assess the severity of AEs and the study intervention 
relationship of AEs based on the patient-reported survey to determine if any action will be needed (eg, reducing dose). An adverse reaction is considered 'serious' when it is life-threatening, causes lasting problems or needs hospital care.

\section{Participant retention and withdrawal}

Strategies to maximise retention will be implemented (ie, survey reminders, emails, phone calls and text messages). A participant will be able to withdraw from the study at any time by signing and returning the withdrawal form. No further data will be collected after withdrawal but previously collected data will be included in the analysis with the permission of the participant. The investigator also may withdraw participants from the study to protect their safety. Participants who drop out and withdraw from the study will not be replaced.

\section{Statistical methods}

\section{Sample Size estimation}

The change in pain VAS at week 12 was used to estimate the sample size using Stata V.14. ${ }^{41}$ The calculation was based on detecting a minimal clinically important difference (MCID) of 15 on a $0-100$ VAS. $^{42}$ The reduction in pain VAS within the given population is approximately 11.3 points (SD 24) ${ }^{43}$ It is hypothesised that a further reduction of at least 15 points would be clinically relevant. To detect a 15-point difference in the change in pain VAS between groups at $80 \%$ power and an alpha level of 0.05 and allowing for $20 \%$ dropout a total of 106 participants would need to be randomised ( 53 per group).

\section{Statistical analysis Plan}

The primary analysis will be according to modified intention to treat, including patients with available outcome data based on their randomised treatment allocation, regardless of compliance.

Analysis will be conducted by the PhD student with the assistance of a biostatistician, who will be blinded to treatment group until the main results are analysed. Demographic characteristics and baseline scores will be presented to assess the comparability of treatment groups using mean and SD for continuous variables (eg, pain, physical function) or medians (range) if the distribution is skewed. Counts with percentages will be presented for categorical variables. The primary analysis for the difference in change of pain VAS score from baseline to 12 weeks between groups will be an Analysis of covariance including adjustment for baseline score to account for possible floor and ceiling effects. A secondary adjusted analysis will also be performed including additional adjustment for baseline and clinical characteristics. Unadjusted differences between groups will also be calculated. Differences in categorical outcome measures will be compared using $\chi^{2}$ tests (or Fisher's exact test if expected cell counts are small). Where normality assumptions are not met, appropriate transformations of the data may be applied or other strategies (use of categories and/or non-parametric tests) may be employed. No interim analyses will be carried out. The two-sided $5 \%$ significance level will be used for all hypothesis tests, with no adjustments for multiple testing.

To assist with the interpretation of the results, we will calculate the minimal clinically important improvement (MCII) for pain and function using the Global Rating of Change (GRC) scale. ${ }^{44}$ The average change score for pain and function of the people who answered the GRC as 'slightly better' will be the cut-off value for MCII. We will also calculate the MCID between groups by using the GRC scale. $^{44}$

\section{Moderator analyses}

Exploratory analyses will be conducted to investigate potential moderators that could influence response to treatment at 12 weeks. Preidentified potential moderators include HOA phenotypes, KLG grade, treatment adherence, participants satisfaction with allocated treatment and technology self-efficacy. The predictability of individual participants' response after a placebo administration will be evaluated using multivariate models (Placebell, Tools4Patient, Jumet Belgium). The performance of those models will be evaluated in Monte-Carlo crossvalidation (repeated random subsampling) in terms of relevant statistics (Pearson's correlation, $\mathrm{R}^{2}$, etc).

Post hoc analysis will include the responder criteria developed by the Outcome Measures in Rheumatology Arthritis Clinical Trials-OARSI. ${ }^{45}$ We will also define as responders the people with a change from baseline in pain intensity beyond the MCII. Logistic regression models adjusted for age, gender, BMI and KLG will be used to compare response between treatment groups.

\section{DATA MANAGEMENT}

All study data will be entered electronically using electronic case report forms built in REDCap which is hosted on The University of Sydney server. REDCap is a secure, web-based application designed to support data capture for research studies. The data will be stored in a reidentifiable format to ensure confidentiality. Backup of reidentifiable information will be kept in password-protected electronic files. Self-monitoring of data entry will be used to maximise data quality.

\section{ETHICS AND DISSEMINATION}

The results of this study will be disseminated in lay summary, infographics, social media, conferences, reports, scientific publications and $\mathrm{PhD}$ thesis. No information which could lead to the identification of a participant will be included in the dissemination of results.

\section{Timelines}

Recruitment commenced in October 2019 and is anticipated to be completed in February 2020. The trial is due 
for completion in June 2020 when all participants will have completed 12-week follow-up.

\section{Twitter Leticia A Deveza @LeticiaDeveza and David Hunter @ProfDavidHunter}

Acknowledgements The authors thank Cherie Burke, Julia Lipski and Peter Sexton for their valuable feedback on the study procedures and relevant documents.

Contributions DH conceived of the study. DH, XL, JE, SR, LAD, TF, SV and AM initiated the study design and XL, SR, TF and SV will facilitate the implementation. $\mathrm{DH}$ is the grant holder. XL will conduct the primary statistical analysis. All authors contributed to the refinement of the study protocol and approved this version. Three patients Cherie Burke, Julia Lipski and Peter Sexton contributed to the pilot study.

Funding The RADIANT study will be funded by an NHMRC Programme Grant (reference number: 1091302).

Competing interests DJH is supported by an NHMRC Practitioner Fellowship and provides consulting advice for Merck Serono, TLC Bio, Tissuegene and Pfizer. Unity Health provides the Sydney Pharmacy School for a researcher's salary for a project led by AM that maintains a database of herb-drug interaction. AM has served as pharmacokinetic consult to BOD Australia on a study investigating cannabidiol bioavailability (ACTRN12618000391279).

Patient consent for publication Not required.

Ethics approval Ethical approval has been obtained from The University of Sydney Human Research Ethics Committee (HREC No 2018/766).

Provenance and peer review Not commissioned; externally peer reviewed.

Open access This is an open access article distributed in accordance with the Creative Commons Attribution Non Commercial (CC BY-NC 4.0) license, which permits others to distribute, remix, adapt, build upon this work non-commercially, and license their derivative works on different terms, provided the original work is properly cited, appropriate credit is given, any changes made indicated, and the use is non-commercial. See: http://creativecommons.org/licenses/by-nc/4.0/.

\section{ORCID iDs}

Xiaogian Liu http://orcid.org/0000-0002-6375-1800

David Hunter http://orcid.org/0000-0003-3197-752X

\section{REFERENCES}

1 GBD 2016 Disease and Injury Incidence and Prevalence Collaborators. Global, regional, and national incidence, prevalence, and years lived with disability for 328 diseases and injuries for 195 countries, 1990-2016: a systematic analysis for the global burden of disease study 2016. Lancet 2017;390:1211-59.

2 Cross M, Smith E, Hoy D, et al. The global burden of hip and knee osteoarthritis: estimates from the global burden of disease 2010 study. Ann Rheum Dis 2014;73:1323-30.

3 Marshall M, Peat G, Nicholls E, et al. Subsets of symptomatic hand osteoarthritis in community-dwelling older adults in the United Kingdom: prevalence, inter-relationships, risk factor profiles and clinical characteristics at baseline and 3-years. Osteoarthritis Cartilage 2013;21:1674-84.

4 Haugen IK, Englund M, Aliabadi P, et al. Prevalence, incidence and progression of hand osteoarthritis in the general population: the Framingham osteoarthritis study. Ann Rheum Dis 2011;70:1581-6.

5 Kodama R, Muraki S, Oka H, et al. Prevalence of hand osteoarthritis and its relationship to hand pain and grip strength in Japan: the third survey of the road study. Mod Rheumatol 2016;26:767-73.

6 Reginster J-YL, Arden NK, Haugen IK, et al. Guidelines for the conduct of pharmacological clinical trials in hand osteoarthritis: consensus of a working group of the European Society on clinical and economic aspects of osteoporosis, osteoarthritis and musculoskeletal diseases (ESCEO). Semin Arthritis Rheum 2018;48:1-8.

7 Kloppenburg M. Hand osteoarthritis-nonpharmacological and pharmacological treatments. Nat Rev Rheumatol 2014;10:242-51.

8 Leung GJ, Rainsford KD, Kean WF. Osteoarthritis of the hand I: aetiology and pathogenesis, risk factors, investigation and diagnosis. J Pharm Pharmacol 2014;66:339-46.

9 Hochberg MC, Altman RD, April KT, et al. American College of rheumatology 2012 recommendations for the use of nonpharmacologic and pharmacologic therapies in osteoarthritis of the hand, hip, and knee. Arthritis Care Res 2012;64:465-74.
10 Kloppenburg M, Kroon FP, Blanco FJ, et al. 2018 update of the EULAR recommendations for the management of hand osteoarthritis. Ann Rheum Dis 2019;78:16-24.

11 Khan MU, Jamshed SQ, Ahmad A, et al. Use of complementary and alternative medicine among osteoarthritic patients: a review. J Clin Diagn Res 2016;10:Je01-6.

12 Corp N, Jordan JL, Croft PR. Justifications for using complementary and alternative medicine reported by persons with musculoskeletal conditions: a narrative literature synthesis. PLoS One 2018;13:e0200879.

13 Zochling J, March L, Lapsley $\mathrm{H}$, et al. Use of complementary medicines for osteoarthritis--a prospective study. Ann Rheum Dis 2004;63:549-54.

14 Basedow M, Runciman WB, March L, et al. Australians with osteoarthritis; the use of and beliefs about complementary and alternative medicines. Complement Ther Clin Pract 2014;20:237-42.

15 Ramsey SD, Spencer AC, Topolski TD, et al. Use of alternative therapies by older adults with osteoarthritis. Arthritis Rheum 2001;45:222-7.

16 Liu X, Machado GC, Eyles JP, et al. Dietary supplements for treating osteoarthritis: a systematic review and meta-analysis. $\mathrm{Br} J$ Sports Med 2018;52:167-75.

17 Liu X, Eyles J, McLachlan AJ, et al. Which supplements can I recommend to my osteoarthritis patients? Rheumatology 2018;57:iv75-87.

18 Liu X, Hunter DJ, Eyles J, et al. Pharmacokinetic assessment of constituents of Boswellia serrata, pine bark extracts, curcumin in combination including methylsulfonylmethane in healthy volunteers. J Pharm Pharmacol 2020;72:121-31.

19 Marks R, Bristol H, Conlon M, et al. Enhancing clinical trials on the Internet: lessons from invest. Clin Cardiol 2001;24:V-17-23.

20 McAlindon T, Formica M, Kabbara K, et al. Conducting clinical trials over the Internet: feasibility study. BMJ 2003;327:484-7.

21 McAlindon T, Formica M, LaValley M, et al. Effectiveness of glucosamine for symptoms of knee osteoarthritis: results from an Internet-based randomized double-blind controlled trial. Am J Med 2004;117:643-9.

22 Bjerkan J, Hedlund M, Hellesø R. Patients' contribution to the development of a web-based plan for integrated care - a participatory design study. Inform Health Soc Care 2015;40:167-84.

23 Joensson K, Melholt C, Hansen J, et al. Listening to the patients: using participatory design in the development of a cardiac telerehabilitation web portal. Mhealth 2019:5:33.

24 Chan A-W, Tetzlaff JM, Altman DG, et al. Spirit 2013 statement: defining standard protocol items for clinical trials. Ann Intern Med 2013;158:200-7.

25 Altman R, Alarcón G, Appelrouth D, et al. The American College of rheumatology criteria for the classification and reporting of osteoarthritis of the hand. Arthritis Rheum 1990;33:1601-10.

26 Kellgren JH, Lawrence JS. Radiological assessment of osteoarthrosis. Ann Rheum Dis 1957;16:494-502.

27 Poole JL. Measures of hand function: arthritis hand function test (AHFT), Australian Canadian osteoarthritis hand index (AUSCAN), Cochin hand function scale, functional index for hand osteoarthritis (FIHOA), grip ability test (GAT), Jebsen hand function test (JHFT), and Michigan hand outcomes questionnaire (MHQ). Arthritis Care Res 2011;63:S189-99.

28 Rohekar G, Pope J. Test-Retest reliability of patient global assessment and physician global assessment in rheumatoid arthritis. $J$ Rheumatol 2009;36:2178-82.

29 Busija L, Pausenberger E, Haines TP, et al. Adult measures of general health and health-related quality of life: medical outcomes study short form 36-Item (SF-36) and short form 12-Item (SF-12) health surveys, Nottingham health profile (NHP), sickness impact profile (SIP), medical outcomes study short form 6D (SF-6D), health Utilities index mark 3 (HUI3), quality of well-being scale (QWB), and assessment of quality of life (AQoL). Arthritis Care Res 2011;63:S383-412.

30 Zhang W, Bansback N, Boonen A, et al. Validity of the work productivity and activity impairment questionnaire--general health version in patients with rheumatoid arthritis. Arthritis Res Ther 2010;12:R177.

31 Voils $\mathrm{Cl}$, Maciejewski ML, Hoyle $\mathrm{RH}$, et al. Initial validation of a self-report measure of the extent of and reasons for medication nonadherence. Med Care 2012;50:1013-9.

32 Vernon MK, Revicki DA, Awad AG, et al. Psychometric evaluation of the medication satisfaction questionnaire (MSQ) to assess satisfaction with antipsychotic medication among schizophrenia patients. Schizophr Res 2010;118:271-8. 
33 Kamper SJ, Maher CG, Mackay G. Global rating of change scales: a review of strengths and weaknesses and considerations for design. $J$ Man Manip Ther 2009;17:163-70.

34 Brislin SJ, Drislane LE, Smith ST, et al. Development and validation of triarchic psychopathy scales from the multidimensional personality questionnaire. Psychol Assess 2015;27:838-51.

35 Brislin SJ, Venables NC, Drislane LE, et al. Further validation of Triarchic psychopathy scales from the multidimensional personality questionnaire: setting the stage for Large-Sample etiological studies. Assessment 2017;24:575-90.

36 Laver K, George S, Ratcliffe J, et al. Measuring technology self efficacy: reliability and construct validity of a modified computer self efficacy scale in a clinical rehabilitation setting. Disabil Rehabil 2012;34:220-7.

37 Marshall M, Jonsson $\mathrm{H}$, Helgadottir GP, et al. Longitudinal validity of using digital hand photographs for assessing hand osteoarthritis progression over 7 years in community-dwelling older adults with hand pain. BMC Musculoskelet Disord 2019;20:484.

38 Jonsson H, Helgadottir GP, Aspelund T, et al. The use of digital Photographs for the diagnosis of hand osteoarthritis: the AGESReykjavik study. BMC Musculoskelet Disord 2012;13:20.

39 Bijsterbosch J, Haugen IK, Malines C, et al. Reliability, sensitivity to change and feasibility of three radiographic scoring methods for hand osteoarthritis. Ann Rheum Dis 2011;70:1465-7.
40 Altman RD, Gold GE. Atlas of individual radiographic features in osteoarthritis, revised. Osteoarthritis Cartilage 2007;15:A1-56.

41 Stata Technical Support. StataCorp. Stata statistical software: release 14. College Station, TX: StataCorp LP, 2015.

42 Tubach F, Ravaud P, Martin-Mola E, et al. Minimum clinically important improvement and patient acceptable symptom state in pain and function in rheumatoid arthritis, ankylosing spondylitis, chronic back pain, hand osteoarthritis, and hip and knee osteoarthritis: results from a prospective multinational study. Arthritis Care Res 2012;64:1699-707.

43 Gabay C, Medinger-Sadowski C, Gascon D, et al. Symptomatic effects of chondroitin 4 and chondroitin 6 sulfate on hand osteoarthritis: a randomized, double-blind, placebo-controlled clinical trial at a single center. Arthritis Rheum 2011;63:3383-91.

44 Copay AG, Subach BR, Glassman SD, et al. Understanding the minimum clinically important difference: a review of concepts and methods. Spine $J$ 2007;7:541-6.

45 Pham T, van der Heijde D, Altman RD, et al. OMERACT-OARSI initiative: osteoarthritis research Society international set of Responder criteria for osteoarthritis clinical trials revisited. Osteoarthritis Cartilage 2004;12:389-99. 